\title{
Testing for Harmonic Regressors
}

\author{
Philip Hans Franses \\ Bert de Groot \\ Rianne Legerstee
}

Econometric Institute, Erasmus University Rotterdam

Econometric Institute Report 2007-04

\begin{abstract}
This paper reports on simulation results for the Wald test for $\alpha_{1}=\alpha_{2}=0$ in the regression model

$$
y_{t}=\mu+\alpha_{1} \cos \left(\frac{2 \pi t}{\kappa}\right)+\alpha_{2} \sin \left(\frac{2 \pi t}{\kappa}\right)+u_{t}
$$

for the case where $\kappa$ is known and for the case where $\kappa$ has to be estimated using nonlinear least squares (NLS). This last situation is not standard, and we therefore provide critical values for further use. A power study shows that choosing inappropriate starting values for $\kappa$ leads to a quick loss of power.
\end{abstract}

Key words: Harmonic regressors, critical values

January 312007

Address for correspondence: Econometric Institute, Erasmus University Rotterdam, P.O. Box 1738, NL-3000 DR Rotterdam, The Netherlands, franses@,few.eur.nl, phone: +31104081273 


\section{Introduction}

Many macroeconomic time series variables, like GDP, unemployment and inflation, experience cycles. Some variables even seem to have more than one such cycle. Cyclical patterns can be associated with short-run business cycles, while others have cycles closer to 30 or $50-60$ years, with the last usually associated with the Kondratieff cycle.

The literature on macroeconomic fluctuations discusses two views on such cycles. The first view assumes that cycles are purely governed by shocks, and, as a consequence, the best model to capture cycles is an autoregression with at least two complex roots. The second view also allows for the effects of shocks, but, at the same time, assumes that part of the cyclical behaviour is deterministic. In this second case, autoregressive models can be augmented with so-called harmonic regressors $\cos \left(\frac{2 \pi t}{\kappa}\right)$ and $\sin \left(\frac{2 \pi t}{\kappa}\right)$, where $t=1,2, \ldots, n$, and $\kappa$ is the period of the cycle. A side effect of this second view on cycles is that forecasts from these models exhibit cyclical behavior as well, while those forecasts from purely autoregressive models converge to the unconditional mean in the long run.

When $\kappa$ is assumed as known and fixed, the Wald test for the joint significance of these two regressors is distributed as $\chi^{2}(2)$. Notice the similarity with tests for seasonal dummies in which case $\kappa$ is associated with cycles within a year, for example. In contrast, when $\kappa$ is unknown and needs to be estimated too, then this Wald test shall not have a $\chi^{2}(2)$ distribution due to the so-called Davies $(1977)^{1}$ problem. Indeed, when the coefficients for $\cos \left(\frac{2 \pi t}{\kappa}\right)$ and $\sin \left(\frac{2 \pi t}{\kappa}\right)$ are equal to zero, under the null hypothesis, the parameter $\kappa$ is not identified.

In this paper we aim to find the critical values for the joint Wald test in case $\kappa$ has to be estimated too. For this purpose we use a range of simulation experiments (with

\footnotetext{
${ }^{1}$ Davies, RB. (1977), Hypothesis testing when the nuisance parameter is present only under the alternative, Biometrika, 64, 247-254.
} 
each time 1000 replications). In Section 3 we show with simulations that the $\chi^{2}$ (2) distribution is useful indeed, when $\kappa$ is known. Next, in the first part of Section 3 we show that when $\kappa$ is unknown this distribution does not hold, although we do notice various similarities for the empirical size fractions across a range of data generating processes (DGPs). This strengthens us to assume that there would be a common distribution across values of $\kappa$ and a range of starting values in the NLS estimation routine. Therefore, in the second part of Section 3 we generate critical values for the Wald test in case $\kappa$ is unknown. In Section 4 we report on the empirical power of the Wald test for unknown $\kappa$ for various starting values in the NLS estimation routine. We see that a proper choice of such a starting value is of substantial importance for the power of the test. In Section 5 we conclude with some practical recommendations.

\section{Simulation results for known $\boldsymbol{\kappa}$}

This section starts with testing for $\alpha_{1}=\alpha_{2}=0$ in the test regression

$$
y_{t}=\mu+\alpha_{1} \cos \left(\frac{2 \pi t}{\kappa}\right)+\alpha_{2} \sin \left(\frac{2 \pi t}{\kappa}\right)+u_{t}
$$

where the DGP is one of the six DGPs in Table 1 (pre-sample observations are set at 0 ). We consider this case for sample sizes 50,100, 200 and 500. And, we fix $\kappa$ at 5 and 20. By including DGPs with autoregressive dynamics we aim to see what the effects are of neglecting such dynamics in the test regression. A priori, we would expect that neglecting such dynamics can lead to too high an empirical size because part of the data may behave as if they have deterministic cycles.

Table 2.1 gives the empirical rejection frequencies in case we use a significance level of $1 \%, 5 \%, 10 \%$ and $20 \%$, where we estimate the parameters in the test regression in (1). In case the DGP is white noise (DGP I), we should expect to see rejection frequencies close to the empirical size and that is what we see indeed (see the boldface rows in Table 2.1). Furthermore, we generally see too high an empirical size in case $\kappa$ is fixed at 20, but never for $\kappa$ is 5 , when the DGP has autoregressive dynamics which are neglected in the test regression. 
Table 2.2 reports on the joint significance of $\alpha_{1}=\alpha_{2}=0$ in the test regression

$$
y_{t}=\mu+\alpha_{1} \cos \left(\frac{2 \pi t}{\kappa}\right)+\alpha_{2} \sin \left(\frac{2 \pi t}{\kappa}\right)+\rho_{1} y_{t-1}+u_{t}
$$

for each of the six DGPs. Looking at the results for DGPs II, III and IV (boldface numbers in Table 2.2), we see that appropriately including lags in the test regression leads to an empirical size that is about equal to the nominal size, certainly when the sample size gets larger. Whether the DGP is non-stationary (DGP IV) does not seem to matter. Neglected second order autoregressive dynamics sometimes leads to a higher size, sometimes to a lower size. The effects of unnecessarily including dynamics (as is the case for DGP I) seem to be irrelevant.

Table 2.3 considers the regression

$$
y_{t}=\mu+\alpha_{1} \cos \left(\frac{2 \pi t}{\kappa}\right)+\alpha_{2} \sin \left(\frac{2 \pi t}{\kappa}\right)+\rho_{1} y_{t-1}+\rho_{2} y_{t-2}+u_{t}
$$

Generally, and similar to Table 2.2, we see that adding too many lags is not harmful for the empirical size of the test, while correctly adding lags (boldface rows) gives the proper size.

\section{Simulation results for unknown $\kappa$}

In this section we repeat the exercise of Section 2, where now the value of $\kappa$ is estimated alongside with the other parameters in (1), (2) or (3). In the second part of this section, we deal with critical values.

\subsection{Non-standard distribution}

We now examine the empirical size of the Wald test for DGPs I to VI for test regressions (1), (2) and (3) in case $\kappa$ is estimated using the NLS routine.

Table 3.1 contains the empirical size values obtained using the same procedure as for Table 2.1, that is using regression (1), but now for unknown $\kappa$. Again we consider the 
cases of $\kappa=5$ and $\kappa=20$, and we take these values as starting values in the NLS routine, which makes sure that the estimation routine converges rapidly. Below in Section 4 we will examine what happens if one takes inappropriate starting values. The cells in Table 3.1 (see the boldface row for DGP I) clearly indicate that the nominal and empirical size are not equal, at least not when the $\chi^{2}(2)$ distribution is assumed (apparently incorrectly). It is clear that the proper asymptotic distribution is further to the right. Further, including not enough lags makes the empirical size to go all over the place.

Tables 3.2 and 3.3 give similar impressions as for Tables 2.2 and 2.3, respectively, that is, including the proper amount of lags (or a little too many, as in Table 3.3) is beneficial for the empirical size. In fact, the main conclusion to be drawn from Table 3.3 is that the empirical size is about equal across DGPs and across values of $\kappa$. At a nominal size of $1 \%$ we get an empirical size of about $5 \%$, at $5 \%$ we get around $20 \%$, at $10 \%$ around $30 \%$ and a nominal $20 \%$ level we get an empirical size of around $50 \%$.

\subsection{Critical values}

The above suggests that there may be an asymptotic distribution of the Wald test for $\alpha_{1}=\alpha_{2}=0$ in test regressions like

$$
y_{t}=\mu+\alpha_{1} \cos \left(\frac{2 \pi t}{\kappa}\right)+\alpha_{2} \sin \left(\frac{2 \pi t}{\kappa}\right)+\rho_{1} y_{t-1}+\ldots .+\rho_{p} y_{t-p}+u_{t}
$$

which is independent of the true but unknown value of $\kappa$. To tabulate the relevant critical values, we generate data from DGP I (white noise) for 1000 observations, and we estimate the parameters of test regression (1), where each time we draw a $\kappa$ from a uniform distribution on 5 to 20 , and where we take the same value as the starting value in the NLS estimation routine. We replicate this simulation 10000 times. The thus obtained critical values appear in Table 4 . The empirical distribution of the test statistic appears in Figure 1. 


\section{The power of the test}

To examine how the Wald test with the proper critical values (those in Table 4) performs in practice, we conclude our set of simulation experiments with a study of the empirical rejection frequency of the test. In Table 5 we give the empirical rejection frequencies for the test for $\alpha_{1}=\alpha_{2}=0$ in the auxiliary regression

$$
y_{t}=\mu+\alpha_{1} \cos \left(\frac{2 \pi t}{\kappa}\right)+\alpha_{2} \sin \left(\frac{2 \pi t}{\kappa}\right)+u_{t}
$$

The DGP is taken as

$$
y_{t}=\mu+\beta \cos \left(\frac{2 \pi t}{\kappa}\right)+\beta \sin \left(\frac{2 \pi t}{\kappa}\right)+\varepsilon_{t}
$$

with $\kappa=5$ or $\kappa=20$, and $\mathrm{N}(0,1)$ errors. The $\beta$ is set at 1 and 10 , where the last case shows visually very clear cycles, while in the first case the noise component predominates.

In the NLS estimation routines we consider a range of starting values. For $\kappa=5$ we take starting values $3,4,4.5,5,5.5,6$ and 7 . The results appear in the first panel of Table 5. First of all, we see that if the estimation routine starts with the correct $\kappa$ value, the empirical power is $100 \%$. Second, we see that once one takes starting values a little off the true values the empirical rejection frequency quickly goes to zero. Our unreported estimation results on the final estimation results for $\kappa$ itself show that these final estimates are usually close to the initial starting value. Hence, even though one might get seemingly plausible estimates for $\kappa$, the Wald test is very informative on their statistical relevance ${ }^{2}$. Note that 4.5 and 5.5 differ a $10 \%$ from the true value of 5 .

The second panel in Table 5 shows that when the cycle period becomes longer (from 5 to 20), the empirical rejection frequency for incorrectly started estimation runs goes to zero much more slowly. Also, but as expected, when the starting values differ only $5 \%$ form the true value (19 and 21 versus 20 ), then one might frequently find the Wald test to reject.

\footnotetext{
${ }^{2}$ There is some literature on the asymptotic distribution of the NLS estimator of $\kappa$, and again this distribution is a non-standard one, see Gallant, A.R. (1982), On the bias in flexible functional forms and an essentially unbiased form, Journal of Econometrics, 15, 211-245.
} 
These findings suggest that if one starts with the proper starting value, one will always find statistical evidence of a deterministic cycle if there is one. However, if one starts with an incorrect starting value, and the cycle period is relatively long, one can also find evidence for cycles that are not truly present in the data.

\section{Conclusion}

The simulation results in the present paper suggest a few useful guidelines for practical work. The first main result is that the Wald test for the joint significance of two conjunct harmonic regressors with unknown period is not distributed as standard $\chi^{2}$. We did not derive the factual asymptotic distribution, which we leave for further work, but we did tabulate practically relevant critical values.

The second result is that one always need to include enough lags in models with known or unknown cycle length, otherwise one will too often find spurious deterministic cycles. Simple standard diagnostic tools will be useful for this purpose.

The third result is that the selection of proper starting values matters a lot. We find that final estimates of cycle periods do not differ a great deal from the initially imposed starting values, but that the Wald test does get smaller quickly for improper starting values. Hence, for practical purposes it seems best to compute a sequence of Wald tests for a range of fixed cycle periods (for which the test is distributed as standard standard $\chi^{2}$ ) and to take that particular period with the largest test value as the starting value for the NLS routine. 
Table 1: Data generating processes

$$
\begin{array}{ll}
\text { I } & y_{t}=\varepsilon_{t} \text { with } \varepsilon_{t} \sim \mathrm{N}(0,1) \\
\text { II } & y_{t}=0.5 y_{t-1}+\varepsilon_{t} \text { with } \varepsilon_{t} \sim \mathrm{N}(0,1) \\
\text { III } & y_{t}=0.9 y_{t-1}+\varepsilon_{t} \text { with } \varepsilon_{t} \sim \mathrm{N}(0,1) \\
\text { IV } & y_{t}=y_{t-1}+\varepsilon_{t} \text { with } \varepsilon_{t} \sim \mathrm{N}(0,1) \\
\text { V } & y_{t}=1.2 y_{t-1}-0.4 y_{t-2}+\varepsilon_{t} \text { with } \varepsilon_{t} \sim \mathrm{N}(0,1) \\
\text { VI } & y_{t}=0.5 y_{t-1}+0.3 y_{t-2}+\varepsilon_{t} \text { with } \varepsilon_{t} \sim \mathrm{N}(0,1)
\end{array}
$$


Table 2.1: Empirical size of Wald test for $\alpha_{1}=\alpha_{2}=0$ in the auxiliary regression

$$
y_{t}=\mu+\alpha_{1} \cos \left(\frac{2 \pi t}{\kappa}\right)+\alpha_{2} \sin \left(\frac{2 \pi t}{\kappa}\right)+u_{t}
$$

where $\kappa$ is fixed at either 5 or 20 .

DGP

\begin{tabular}{lllllllll} 
Nominal size: $1 \%$ & $\begin{array}{l}2 \\
\text { N } 50\end{array}$ & \multicolumn{3}{c}{$n=100$} & \multicolumn{2}{c}{$n=200$} & \multicolumn{3}{c}{$n=500$} \\
& $\kappa=5$ & $\kappa=20$ & $\kappa=5$ & $\kappa=20$ & $\kappa=5$ & $\kappa=20$ & $\kappa=5$ & $\kappa=20$ \\
I & $\mathbf{1 . 5}$ & $\mathbf{1 . 4}$ & $\mathbf{1 . 3}$ & $\mathbf{0 . 9}$ & $\mathbf{0 . 8}$ & $\mathbf{1 . 3}$ & $\mathbf{1 . 5}$ & $\mathbf{1 . 1}$ \\
II & 0.2 & 19.7 & 0.5 & 19.4 & 0.3 & 18.6 & 0.5 & 17.6 \\
III & 0.0 & 34.2 & 0.0 & 21.7 & 0.0 & 16.5 & 0.0 & 11.1 \\
IV & 0.0 & 18.8 & 0.0 & 5.6 & 0.0 & 0.9 & 0.0 & 0.0 \\
V & 0.0 & 48.4 & 0.0 & 40.9 & 0.0 & 37.6 & 0.0 & 36.7 \\
VI & 0.0 & 30.9 & 0.0 & 22.6 & 0.0 & 17.4 & 0.0 & 16.5
\end{tabular}

Nominal size: $5 \% \quad n=50 \quad n=100 \quad n=200 \quad n=500$

$\kappa=5 \quad \kappa=20 \quad \kappa=5 \quad \kappa=20 \quad \kappa=5 \quad \kappa=20 \quad \kappa=5 \quad \kappa=20$

$\begin{array}{lllllllll}\text { I } & \mathbf{6 . 2} & \mathbf{5 . 0} & \mathbf{6 . 2} & \mathbf{4 . 2} & \mathbf{5 . 5} & \mathbf{5 . 4} & \mathbf{4 . 7} & \mathbf{6 . 1} \\ \text { II } & 3.3 & 34.7 & 2.9 & 36.2 & 3.4 & 34.1 & 2.3 & 30.4 \\ \text { III } & 0.1 & 47.4 & 0.0 & 35.4 & 0.0 & 30.4 & 0.0 & 21.8 \\ \text { IV } & 0.0 & 30.0 & 0.0 & 14.5 & 0.0 & 2.8 & 0.0 & 0.1 \\ \text { V } & 0.1 & 59.8 & 0.1 & 54.4 & 0.0 & 52.6 & 0.0 & 50.8 \\ \text { VI } & 0.3 & 44.8 & 0.0 & 37.6 & 0.0 & 32.0 & 0.0 & 30.0\end{array}$

\begin{tabular}{|c|c|c|c|c|c|c|c|c|}
\hline \multirow[t]{2}{*}{ Nominal size: $10 \%$} & \multicolumn{2}{|c|}{$n=50$} & \multicolumn{2}{|c|}{$n=100$} & \multicolumn{2}{|c|}{$n=200$} & \multicolumn{2}{|c|}{$n=500$} \\
\hline & $\kappa=5$ & $\kappa=20$ & $\kappa=5$ & $\kappa=20$ & $\kappa=5$ & $\kappa=20$ & $\kappa=5$ & $\kappa=20$ \\
\hline I & 10.5 & 11.0 & 10.6 & 8.8 & 11.3 & 10.0 & 10.0 & 10.9 \\
\hline II & 7.5 & 44.9 & 6.7 & 45.8 & 7.5 & 43.2 & 6.4 & 40.0 \\
\hline III & 0.4 & 56.3 & 0.1 & 45.9 & 0.1 & 39.8 & 0.0 & 31.5 \\
\hline IV & 0.0 & 37.9 & 0.0 & 21.5 & 0.0 & 5.6 & 0.0 & 0.3 \\
\hline V & 0.3 & 68.0 & 0.1 & 64.3 & 0.0 & 60.7 & 0.0 & 59.0 \\
\hline VI & 0.5 & 54.0 & 0.4 & 48.0 & 0.0 & 40.9 & 0.0 & 41.5 \\
\hline
\end{tabular}

\begin{tabular}{|c|c|c|c|c|c|c|c|c|}
\hline \multirow{2}{*}{ Nominal size: $20 \%$} & \multicolumn{2}{|c|}{$n=50$} & \multicolumn{2}{|c|}{$n=100$} & \multicolumn{2}{|c|}{$n=200$} & \multicolumn{2}{|c|}{$n=500$} \\
\hline & $\kappa=5$ & $\kappa=20$ & $\kappa=5$ & $\kappa=20$ & $\kappa=5$ & $\kappa=20$ & $\kappa=5$ & $\kappa=20$ \\
\hline I & 20.1 & 22.1 & 22.1 & 18.7 & 22.9 & 18.3 & 21.2 & 20.3 \\
\hline II & 16.7 & 57.2 & 14.8 & 58.2 & 16.5 & 58.0 & 14.5 & 52.8 \\
\hline III & 1.7 & 66.9 & 0.2 & 56.9 & 0.2 & 50.0 & 0.0 & 47.5 \\
\hline IV & 0.4 & 47.4 & 0.0 & 34.1 & 0.0 & 12.6 & 0.0 & 1.5 \\
\hline V & 1.6 & 78.0 & 0.7 & 73.0 & 0.1 & 69.6 & 0.3 & 68.7 \\
\hline VI & 2.8 & 64.9 & 1.5 & 59.7 & 0.8 & 51.9 & 0.7 & 53.3 \\
\hline
\end{tabular}


Table 2.2: Empirical size of Wald test for $\alpha_{1}=\alpha_{2}=0$ in the auxiliary regression

$$
y_{t}=\mu+\alpha_{1} \cos \left(\frac{2 \pi t}{\kappa}\right)+\alpha_{2} \sin \left(\frac{2 \pi t}{\kappa}\right)+\rho_{1} y_{t-1}+u_{t}
$$

where $\kappa$ is fixed at either 5 or 20 .

DGP

\begin{tabular}{llllllllll} 
Nominal size: $1 \%$ & \multicolumn{1}{c}{$\begin{array}{l}n=50 \\
\kappa=5\end{array}$} & $\kappa=20$ & $\kappa=5$ & $\kappa=20$ & $\kappa=5$ & $\kappa=20$ & $\kappa=5$ & $\kappa=20$ \\
& & & & & & & & \\
I & 2.1 & 1.9 & 1.8 & 1.6 & 1.5 & 1.3 & 0.8 & 0.9 \\
II & $\mathbf{2 . 1}$ & $\mathbf{3 . 7}$ & $\mathbf{1 . 6}$ & $\mathbf{1 . 6}$ & $\mathbf{0 . 7}$ & $\mathbf{0 . 7}$ & $\mathbf{0 . 5}$ & $\mathbf{0 . 7}$ \\
III & $\mathbf{1 . 3}$ & $\mathbf{3 . 0}$ & $\mathbf{0 . 6}$ & $\mathbf{1 . 7}$ & $\mathbf{1 . 4}$ & $\mathbf{1 . 4}$ & $\mathbf{0 . 7}$ & $\mathbf{1 . 1}$ \\
IV & $\mathbf{1 . 3}$ & $\mathbf{3 . 8}$ & $\mathbf{1 . 3}$ & $\mathbf{1 . 5}$ & $\mathbf{0 . 6}$ & $\mathbf{1 . 1}$ & $\mathbf{1 . 4}$ & $\mathbf{0 . 6}$ \\
V & 1.7 & 9.4 & 1.9 & 7.2 & 2.1 & 6.8 & 1.8 & 6.7 \\
VI & 0.3 & 3.8 & 0.2 & 1.0 & 0.0 & 0.7 & 0.1 & 0.2
\end{tabular}

Nominal size: $5 \% \quad n=50 \quad n=100 \quad n=200 \quad n=500$

$\kappa=5 \quad \kappa=20 \quad \kappa=5 \quad \kappa=20 \quad \kappa=5 \quad \kappa=20 \quad \kappa=5 \quad \kappa=20$

$\begin{array}{lllllllll}\text { I } & 7.4 & 7.5 & 6.3 & 6.4 & 5.7 & 6.1 & 4.7 & 6.9 \\ \text { II } & \mathbf{6 . 9} & \mathbf{9 . 9} & \mathbf{5 . 4} & \mathbf{6 . 1} & \mathbf{4 . 7} & \mathbf{5 . 5} & \mathbf{4 . 3} & \mathbf{6 . 0} \\ \text { III } & \mathbf{5 . 0} & \mathbf{1 0 . 4} & \mathbf{4 . 3} & \mathbf{6 . 3} & \mathbf{4 . 5} & \mathbf{5 . 8} & \mathbf{5 . 6} & \mathbf{4 . 5} \\ \text { IV } & \mathbf{5 . 6} & \mathbf{1 0 . 2} & \mathbf{4 . 7} & \mathbf{7 . 1} & \mathbf{5 . 7} & \mathbf{5 . 7} & \mathbf{6 . 3} & \mathbf{3 . 5} \\ \text { V } & 7.2 & 20.8 & 7.3 & 19.8 & 8.5 & 17.6 & 7.4 & 18.0 \\ \text { VI } & 1.7 & 11.7 & 0.7 & 4.2 & 0.4 & 2.4 & 0.9 & 2.6\end{array}$

Nominal size: $10 \% \quad n=50 \quad n=100 \quad n=200 \quad n=500$

$\kappa=5 \quad \kappa=20 \quad \kappa=5 \quad \kappa=20 \quad \kappa=5 \quad \kappa=20 \quad \kappa=5 \quad \kappa=20$

$\begin{array}{lllllllll}\text { I } & 11.2 & 13.7 & 12.3 & 11.8 & 11.6 & 12.0 & 9.7 & 11.9 \\ \text { II } & \mathbf{1 1 . 0} & \mathbf{1 6 . 8} & \mathbf{9 . 8} & \mathbf{1 2 . 4} & \mathbf{1 1 . 4} & \mathbf{1 1 . 6} & \mathbf{9 . 2} & \mathbf{1 0 . 8} \\ \text { III } & \mathbf{8 . 9} & \mathbf{1 7 . 5} & \mathbf{8 . 6} & \mathbf{1 2 . 2} & \mathbf{1 0 . 0} & \mathbf{1 0 . 6} & \mathbf{9 . 5} & \mathbf{8 . 3} \\ \text { IV } & \mathbf{1 0 . 3} & \mathbf{1 7 . 2} & \mathbf{1 0 . 3} & \mathbf{1 3 . 5} & \mathbf{1 0 . 5} & \mathbf{1 1 . 2} & \mathbf{1 1 . 1} & \mathbf{7 . 8} \\ \text { V } & 12.7 & 28.8 & 13.1 & 29.0 & 13.2 & 27.5 & 12.6 & 24.5 \\ \text { VI } & 4.4 & 17.7 & 2.2 & 9.0 & 2.2 & 5.6 & 2.1 & 6.1\end{array}$

Nominal size: $20 \% \quad n=50 \quad n=100 \quad n=200 \quad n=500$

$\kappa=5 \quad \kappa=20 \quad \kappa=5 \quad \kappa=20 \quad \kappa=5 \quad \kappa=20 \quad \kappa=5 \quad \kappa=20$

$\begin{array}{lllllllll}\text { I } & 20.9 & 23.8 & 22.6 & 21.9 & 21.3 & 23.0 & 20.7 & 22.0 \\ \text { II } & \mathbf{2 1 . 7} & \mathbf{2 8 . 5} & \mathbf{1 9 . 4} & \mathbf{2 3 . 9} & \mathbf{2 0 . 6} & \mathbf{2 2 . 3} & \mathbf{1 8 . 7} & \mathbf{2 1 . 5} \\ \text { III } & \mathbf{1 7 . 9} & \mathbf{3 0 . 4} & \mathbf{1 8 . 1} & \mathbf{2 1 . 8} & \mathbf{2 1 . 2} & \mathbf{2 2 . 0} & \mathbf{1 8 . 9} & \mathbf{1 8 . 3} \\ \text { IV } & \mathbf{2 0 . 2} & \mathbf{2 8 . 3} & \mathbf{1 8 . 5} & \mathbf{2 4 . 1} & \mathbf{1 9 . 0} & \mathbf{2 0 . 6} & \mathbf{2 1 . 1} & \mathbf{1 8 . 7} \\ \text { V } & 25.2 & 43.7 & 23.0 & 41.8 & 22.9 & 40.2 & 22.3 & 38.9 \\ \text { VI } & 10.5 & 28.9 & 7.6 & 19.7 & 6.7 & 14.7 & 6.4 & 13.3\end{array}$


Table 2.3: Empirical size of Wald test for $\alpha_{1}=\alpha_{2}=0$ in the auxiliary regression

$$
y_{t}=\mu+\alpha_{1} \cos \left(\frac{2 \pi t}{\kappa}\right)+\alpha_{2} \sin \left(\frac{2 \pi t}{\kappa}\right)+\rho_{1} y_{t-1}+\rho_{2} y_{t-2}+u_{t}
$$

where $\kappa$ is fixed at either 5 or 20 .

DGP

\begin{tabular}{lllllllll} 
Nominal size: $1 \%$ & \multicolumn{1}{c}{$\begin{array}{l}n=50 \\
\kappa=5\end{array}$} & $\kappa=20$ & $\kappa=5$ & $\kappa=20$ & $\kappa=5$ & $\kappa=20$ & $\kappa=5$ & $\kappa=20$ \\
& & & & & & & & \\
I & 2.2 & 2.4 & 0.8 & 1.9 & 1.0 & 1.0 & 1.1 & 0.9 \\
II & 1.2 & 4.4 & 1.2 & 1.6 & 1.2 & 1.5 & 0.7 & 0.6 \\
III & 0.9 & 3.3 & 1.1 & 2.0 & 0.5 & 1.8 & 0.9 & 1.0 \\
IV & 1.2 & 2.6 & 1.4 & 1.5 & 1.3 & 1.0 & 1.0 & 0.5 \\
V & $\mathbf{1 . 1}$ & $\mathbf{5 . 1}$ & $\mathbf{1 . 1}$ & $\mathbf{2 . 3}$ & $\mathbf{1 . 0}$ & $\mathbf{1 . 6}$ & $\mathbf{0 . 5}$ & $\mathbf{2 . 0}$ \\
VI & $\mathbf{1 . 4}$ & $\mathbf{4 . 9}$ & $\mathbf{1 . 4}$ & $\mathbf{2 . 2}$ & $\mathbf{1 . 4}$ & $\mathbf{1 . 0}$ & $\mathbf{1 . 0}$ & $\mathbf{1 . 3}$
\end{tabular}

Nominal size: $5 \% \quad n=50 \quad n=100 \quad n=200 \quad n=500$

$\kappa=5 \quad \kappa=20 \quad \kappa=5 \quad \kappa=20 \quad \kappa=5 \quad \kappa=20 \quad \kappa=5 \quad \kappa=20$

$\begin{array}{lllllllll}\text { I } & 5.8 & 8.4 & 4.4 & 7.7 & 5.2 & 5.0 & 4.2 & 4.4 \\ \text { II } & 4.8 & 10.5 & 5.1 & 6.9 & 5.8 & 4.8 & 5.5 & 4.2 \\ \text { III } & 4.8 & 9.2 & 5.3 & 5.9 & 4.6 & 6.0 & 5.7 & 6.1 \\ \text { IV } & 4.9 & 9.8 & 6.4 & 6.8 & 5.1 & 4.8 & 5.2 & 5.3 \\ \text { V } & \mathbf{4 . 8} & \mathbf{1 4 . 7} & \mathbf{4 . 4} & \mathbf{9 . 3} & \mathbf{4 . 6} & \mathbf{6 . 7} & \mathbf{4 . 5} & \mathbf{7 . 1} \\ \text { VI } & \mathbf{4 . 9} & \mathbf{1 2 . 1} & \mathbf{5 . 8} & \mathbf{6 . 9} & \mathbf{4 . 7} & \mathbf{6 . 0} & \mathbf{4 . 4} & \mathbf{5 . 2}\end{array}$

Nominal size: $10 \% \quad n=50 \quad n=100 \quad n=200 \quad n=500$

$\kappa=5 \quad \kappa=20 \quad \kappa=5 \quad \kappa=20 \quad \kappa=5 \quad \kappa=20 \quad \kappa=5 \quad \kappa=20$

$\begin{array}{lllllllll}\text { I } & 10.8 & 14.1 & 10.6 & 12.7 & 10.2 & 10.7 & 9.0 & 8.4 \\ \text { II } & 8.9 & 17.9 & 11.2 & 12.1 & 10.9 & 9.4 & 10.7 & 8.6 \\ \text { III } & 9.1 & 16.2 & 10.1 & 11.6 & 8.9 & 11.6 & 9.7 & 10.7 \\ \text { IV } & 9.8 & 16.2 & 10.1 & 11.6 & 10.5 & 10.0 & 9.4 & 11.4 \\ \text { V } & \mathbf{1 0 . 0} & \mathbf{2 2 . 3} & \mathbf{8 . 2} & \mathbf{1 5 . 7} & \mathbf{8 . 4} & \mathbf{1 2 . 8} & \mathbf{1 1 . 4} & \mathbf{1 2 . 4} \\ \text { VI } & \mathbf{1 0 . 1} & \mathbf{2 0 . 8} & \mathbf{1 0 . 8} & \mathbf{1 2 . 6} & \mathbf{8 . 6} & \mathbf{1 1 . 0} & \mathbf{9 . 9} & \mathbf{9 . 3}\end{array}$

Nominal size: $20 \% \quad n=50 \quad n=100 \quad n=200 \quad n=500$

$\kappa=5 \quad \kappa=20 \quad \kappa=5 \quad \kappa=20 \quad \kappa=5 \quad \kappa=20 \quad \kappa=5 \quad \kappa=20$

$\begin{array}{lllllllll}\text { I } & 21.0 & 25.8 & 19.7 & 24.0 & 19.8 & 22.3 & 18.5 & 20.3 \\ \text { II } & 19.4 & 29.8 & 21.3 & 21.9 & 19.5 & 19.0 & 21.4 & 18.0 \\ \text { III } & 19.4 & 28.2 & 20.3 & 22.8 & 18.9 & 21.7 & 18.3 & 21.8 \\ \text { IV } & 19.8 & 28.2 & 19.1 & 21.1 & 21.0 & 17.8 & 19.1 & 20.2 \\ \text { V } & \mathbf{2 2 . 0} & \mathbf{3 4 . 4} & \mathbf{1 8 . 6} & \mathbf{2 6 . 1} & \mathbf{1 8 . 6} & \mathbf{2 3 . 6} & \mathbf{2 0 . 3} & \mathbf{2 2 . 9} \\ \text { VI } & \mathbf{1 7 . 8} & \mathbf{3 3 . 3} & \mathbf{2 0 . 6} & \mathbf{2 3 . 2} & \mathbf{1 9 . 8} & \mathbf{2 0 . 8} & \mathbf{2 0 . 3} & \mathbf{2 0 . 5}\end{array}$


Table 3.1: Empirical size of Wald test for $\alpha_{1}=\alpha_{2}=0$ in the auxiliary regression

$$
y_{t}=\mu+\alpha_{1} \cos \left(\frac{2 \pi t}{\kappa}\right)+\alpha_{2} \sin \left(\frac{2 \pi t}{\kappa}\right)+u_{t}
$$

where $\kappa$ is estimated using Nonlinear Least Squares, with starting values 5 or 20.

DGP

\begin{tabular}{|c|c|c|c|c|c|c|c|c|}
\hline \multirow[t]{2}{*}{ Nominal size: $1 \%$} & \multicolumn{2}{|c|}{$n=50$} & \multicolumn{2}{|c|}{$n=100$} & \multicolumn{2}{|c|}{$n=200$} & \multicolumn{2}{|c|}{$n=500$} \\
\hline & $\kappa=5$ & $\kappa=20$ & $\kappa=5$ & $\kappa=20$ & $\kappa=5$ & $\kappa=20$ & $\kappa=5$ & $\kappa=20$ \\
\hline I & 6.1 & 6.0 & 5.6 & 6.3 & 5.0 & 5.2 & 4.0 & 5.8 \\
\hline II & 4.6 & 50.3 & 2.1 & 48.0 & 2.3 & 43.0 & 1.6 & 44.4 \\
\hline III & 0.1 & 70.5 & 0.0 & 51.7 & 0.0 & 41.9 & 0.0 & 34.3 \\
\hline IV & 0.0 & 57.6 & 0.1 & 21.7 & 0.0 & 3.9 & 0.0 & 0.1 \\
\hline $\mathrm{V}$ & 0.0 & 83.0 & 0.1 & 75.1 & 0.0 & 72.6 & 0.0 & 71.4 \\
\hline VI & 0.7 & 66.8 & 0.0 & 52.2 & 0.0 & 46.5 & 0.0 & 43.2 \\
\hline
\end{tabular}

\begin{tabular}{|c|c|c|c|c|c|c|c|c|}
\hline \multirow[t]{2}{*}{ Nominal size: $5 \%$} & \multicolumn{2}{|c|}{$n=50$} & \multicolumn{2}{|c|}{$n=100$} & \multicolumn{2}{|c|}{$n=200$} & \multicolumn{2}{|c|}{$n=500$} \\
\hline & $\kappa=5$ & $\kappa=20$ & $\kappa=5$ & $\kappa=20$ & $\kappa=5$ & $\kappa=20$ & $\kappa=5$ & $\kappa=20$ \\
\hline I & 18.8 & 20.6 & 20.0 & 19.4 & 18.1 & 20.5 & 15.4 & 16.9 \\
\hline II & 14.5 & 69.6 & 11.0 & 67.2 & 10.2 & 64.6 & 8.4 & 66.5 \\
\hline III & 0.3 & 80.7 & 0.0 & 68.7 & 0.0 & 60.1 & 0.0 & 54.9 \\
\hline IV & 0.1 & 70.4 & 0.1 & 35.7 & 0.0 & 10.1 & 0.0 & 0.7 \\
\hline V & 0.2 & 91.3 & 0.1 & 86.9 & 0.0 & 85.0 & 0.0 & 84.3 \\
\hline VI & 2.7 & 77.8 & 0.4 & 71.9 & 0.0 & 64.5 & 0.0 & 63.3 \\
\hline
\end{tabular}

\begin{tabular}{|c|c|c|c|c|c|c|c|c|}
\hline \multirow[t]{2}{*}{ Nominal size: $10 \%$} & \multicolumn{2}{|c|}{$n=50$} & \multicolumn{2}{|c|}{$n=100$} & \multicolumn{2}{|c|}{$n=200$} & \multicolumn{2}{|c|}{$n=500$} \\
\hline & $\kappa=5$ & $\kappa=20$ & $\kappa=5$ & $\kappa=20$ & $\kappa=5$ & $\kappa=20$ & $\kappa=5$ & $\kappa=20$ \\
\hline I & 30.9 & 32.8 & 33.8 & 32.1 & 32.5 & 32.8 & 28.1 & 29.9 \\
\hline II & 23.7 & 79.6 & 21.1 & 76.2 & 18.7 & 75.8 & 17.4 & 76.1 \\
\hline III & 1.6 & 86.2 & 0.3 & 77.3 & 0.0 & 70.8 & 0.0 & 66.8 \\
\hline IV & 0.1 & 77.6 & 0.1 & 46.1 & 0.0 & 16.7 & 0.0 & 2.6 \\
\hline V & 2.4 & 94.4 & 0.7 & 90.4 & 0.2 & 90.0 & 0.2 & 88.7 \\
\hline VI & 4.9 & 83.7 & 2.2 & 80.6 & 0.4 & 75.3 & 0.4 & 14.4 \\
\hline
\end{tabular}

\begin{tabular}{|c|c|c|c|c|c|c|c|c|}
\hline \multirow[t]{2}{*}{ Nominal size: $20 \%$} & \multicolumn{2}{|c|}{$n=50$} & \multicolumn{2}{|c|}{$n=100$} & \multicolumn{2}{|c|}{$n=200$} & \multicolumn{2}{|c|}{$n=500$} \\
\hline & $\kappa=5$ & $\kappa=20$ & $\kappa=5$ & $\kappa=20$ & $\kappa=5$ & $\kappa=20$ & $\kappa=5$ & $\kappa=20$ \\
\hline I & 48.6 & 51.4 & 51.9 & 46.1 & 49.0 & 50.7 & 49.4 & 48.6 \\
\hline II & 41.3 & 89.7 & 37.3 & 86.3 & 37.7 & 87.2 & 37.3 & 86.3 \\
\hline III & 5.0 & 92.3 & 1.7 & 86.0 & 0.4 & 83.1 & 0.0 & 81.0 \\
\hline IV & 1.6 & 85.3 & 0.3 & 59.1 & 0.0 & 29.2 & 0.0 & 5.4 \\
\hline $\mathrm{V}$ & 8.3 & 97.1 & 2.8 & 94.0 & 1.8 & 94.4 & 1.3 & 92.4 \\
\hline VI & 11.5 & 89.8 & 6.0 & 89.0 & 3.4 & 86.3 & 2.5 & 86.1 \\
\hline
\end{tabular}


Table 3.2: Empirical size of Wald test for $\alpha_{1}=\alpha_{2}=0$ in the auxiliary regression

$$
y_{t}=\mu+\alpha_{1} \cos \left(\frac{2 \pi t}{\kappa}\right)+\alpha_{2} \sin \left(\frac{2 \pi t}{\kappa}\right)+\rho_{1} y_{t-1}+u_{t}
$$

where $\kappa$ is estimated using Nonlinear Least Squares, with starting values 5 or 20.

DGP

\begin{tabular}{|c|c|c|c|c|c|c|c|c|}
\hline \multirow[t]{2}{*}{ Nominal size: $1 \%$} & \multicolumn{2}{|c|}{$n=50$} & \multicolumn{2}{|c|}{$n=100$} & \multicolumn{2}{|c|}{$n=200$} & \multicolumn{2}{|c|}{$n=500$} \\
\hline & $\kappa=5$ & $\kappa=20$ & $\kappa=5$ & $\kappa=20$ & $\kappa=5$ & $\kappa=20$ & $\kappa=5$ & $\kappa=20$ \\
\hline I & 8.8 & 6.9 & 6.4 & 6.2 & 5.9 & 6.5 & 5.6 & 3.9 \\
\hline II & 7.9 & 11.6 & 6.7 & 7.8 & 6.2 & 5.4 & 3.8 & 4.0 \\
\hline III & 4.4 & 20.5 & 4.1 & 8.8 & 4.4 & 6.2 & 5.0 & 4.1 \\
\hline IV & 5.4 & 14.1 & 3.9 & 6.0 & 4.6 & 5.2 & 4.8 & 4.2 \\
\hline $\mathrm{V}$ & 8.8 & 29.5 & 7.3 & 27.4 & 7.5 & 24.5 & 7.6 & 22.5 \\
\hline VI & 0.6 & 26.0 & 0.9 & 7.2 & 0.5 & 3.2 & 0.5 & 2.2 \\
\hline \multirow[t]{2}{*}{ Nominal size: $5 \%$} & \multicolumn{2}{|c|}{$n=50$} & \multicolumn{2}{|c|}{$n=100$} & \multicolumn{2}{|c|}{$n=200$} & \multicolumn{2}{|c|}{$n=500$} \\
\hline & $\kappa=5$ & $\kappa=20$ & $\kappa=5$ & $\kappa=20$ & $\kappa=5$ & $\kappa=20$ & $\kappa=5$ & $\kappa=20$ \\
\hline I & 24.1 & 23.8 & 22.0 & 20.1 & 20.0 & 21.7 & 18.3 & 18.0 \\
\hline II & 22.7 & 32.4 & 20.6 & 25.2 & 19.3 & 20.9 & 19.2 & 18.0 \\
\hline III & 17.5 & 41.5 & 17.8 & 24.5 & 16.0 & 20.7 & 17.2 & 18.2 \\
\hline IV & 19.0 & 33.0 & 17.8 & 19.4 & 18.0 & 16.9 & 18.2 & 14.4 \\
\hline V & 21.8 & 52.6 & 23.4 & 48.0 & 22.8 & 47.3 & 23.0 & 45.0 \\
\hline VI & 5.2 & 42.7 & 4.3 & 20.9 & 3.7 & 12.1 & 3.7 & 10.6 \\
\hline
\end{tabular}

\begin{tabular}{|c|c|c|c|c|c|c|c|c|}
\hline \multirow[t]{2}{*}{ Nominal size: $10 \%$} & \multicolumn{2}{|c|}{$n=50$} & \multicolumn{2}{|c|}{$n=100$} & \multicolumn{2}{|c|}{$n=200$} & \multicolumn{2}{|c|}{$n=500$} \\
\hline & $\kappa=5$ & $\kappa=20$ & $\kappa=5$ & $\kappa=20$ & $\kappa=5$ & $\kappa=20$ & $\kappa=5$ & $\kappa=20$ \\
\hline I & 34.9 & 37.7 & 35.2 & 33.3 & 31.6 & 32.8 & 32.2 & 30.9 \\
\hline II & 37.6 & 46.2 & 32.8 & 39.1 & 30.8 & 33.7 & 30.2 & 31.5 \\
\hline III & 31.1 & 55.3 & 30.2 & 36.9 & 28.0 & 34.1 & 29.5 & 30.8 \\
\hline IV & 30.9 & 48.3 & 29.7 & 33.2 & 29.3 & 29.3 & 29.9 & 27.1 \\
\hline V & 36.5 & 65.7 & 38.8 & 63.0 & 35.2 & 59.5 & 36.8 & 59.6 \\
\hline VI & 10.9 & 53.3 & 9.8 & 31.7 & 9.8 & 22.4 & 9.0 & 20.5 \\
\hline
\end{tabular}

\begin{tabular}{lllllllll} 
Nominal size: $20 \%$ & \multicolumn{1}{c}{$\begin{array}{l}20 \\
\end{array}$} & \multicolumn{3}{c}{$n=100$} & \multicolumn{2}{c}{$n=200$} & \multicolumn{2}{c}{$n=500$} \\
& $\kappa=5$ & $\kappa=20$ & $\kappa=5$ & $\kappa=20$ & $\kappa=5$ & $\kappa=20$ & $\kappa=5$ & $\kappa=20$ \\
I & & & & & & & & \\
II & 55.2 & 55.7 & 53.6 & 52.8 & 51.2 & 50.2 & 52.1 & 51.8 \\
III & 54.4 & 65.8 & 53.1 & 57.6 & 50.4 & 53.4 & 50.9 & 50.0 \\
IV & $\mathbf{4 9 . 9}$ & $\mathbf{7 0 . 4}$ & $\mathbf{4 7 . 5}$ & $\mathbf{5 8 . 3}$ & $\mathbf{4 6 . 4}$ & $\mathbf{5 1 . 2}$ & $\mathbf{5 0 . 6}$ & $\mathbf{4 9 . 9}$ \\
V & $\mathbf{4 8 . 1}$ & $\mathbf{6 2 . 6}$ & $\mathbf{4 9 . 0}$ & $\mathbf{5 3 . 6}$ & $\mathbf{4 8 . 3}$ & $\mathbf{4 7 . 7}$ & $\mathbf{4 8 . 6}$ & $\mathbf{4 4 . 2}$ \\
VI & $\mathbf{5 2 . 8}$ & $\mathbf{7 9 . 9}$ & $\mathbf{5 6 . 0}$ & $\mathbf{7 6 . 1}$ & $\mathbf{5 5 . 6}$ & $\mathbf{7 4 . 0}$ & $\mathbf{5 6 . 7}$ & $\mathbf{7 4 . 8}$ \\
& 24.1 & 68.5 & 23.6 & 48.2 & 25.8 & 41.7 & 22.3 & 40.0
\end{tabular}


Table 3.3: Empirical size of Wald test for $\alpha_{1}=\alpha_{2}=0$ in the auxiliary regression

$$
y_{t}=\mu+\alpha_{1} \cos \left(\frac{2 \pi t}{\kappa}\right)+\alpha_{2} \sin \left(\frac{2 \pi t}{\kappa}\right)+\rho_{1} y_{t-1}+\rho_{2} y_{t-2}+u_{t}
$$

where $\kappa$ is estimated using Nonlinear Least Squares, with starting values 5 or 20 .

DGP

\begin{tabular}{|c|c|c|c|c|c|c|c|c|}
\hline \multirow[t]{2}{*}{ Nominal size: $1 \%$} & \multicolumn{2}{|c|}{$n=50$} & \multicolumn{2}{|c|}{$n=100$} & \multicolumn{2}{|c|}{$n=200$} & \multicolumn{2}{|c|}{$n=500$} \\
\hline & $\kappa=5$ & $\kappa=20$ & $\kappa=5$ & $\kappa=20$ & $\kappa=5$ & $\kappa=20$ & $\kappa=5$ & $\kappa=20$ \\
\hline I & 6.7 & 11.8 & 5.2 & 7.1 & 4.9 & 7.2 & 4.8 & 5.6 \\
\hline II & 5.0 & 15.5 & 4.1 & 8.9 & 3.7 & 5.7 & 3.1 & 5.1 \\
\hline III & 4.3 & 20.6 & 4.2 & 8.4 & 4.1 & 6.0 & 4.6 & 4.8 \\
\hline IV & 4.7 & 14.2 & 5.7 & 7.0 & 4.4 & 5.0 & 5.8 & 5.5 \\
\hline V & 6.0 & 22.4 & 6.0 & 11.1 & 4.0 & 6.3 & 4.5 & 6.1 \\
\hline VI & 4.1 & 18.7 & 3.8 & 8.7 & 4.6 & 7.0 & 4.4 & 5.3 \\
\hline \multirow[t]{2}{*}{ Nominal size: $5 \%$} & \multicolumn{2}{|c|}{$n=50$} & \multicolumn{2}{|c|}{$n=100$} & \multicolumn{2}{|c|}{$n=200$} & \multicolumn{2}{|c|}{$n=500$} \\
\hline & $\kappa=5$ & $\kappa=20$ & $\kappa=5$ & $\kappa=20$ & $\kappa=5$ & $\kappa=20$ & $\kappa=5$ & $\kappa=20$ \\
\hline I & 22.0 & 29.9 & 19.4 & 24.8 & 18.6 & 21.8 & 20.4 & 19.9 \\
\hline II & 18.2 & 36.0 & 17.9 & 26.9 & 17.0 & 21.6 & 15.3 & 20.2 \\
\hline III & 18.4 & 43.9 & 18.7 & 24.4 & 17.0 & 19.1 & 16.1 & 16.7 \\
\hline IV & 18.4 & 31.2 & 18.1 & 21.3 & 17.9 & 18.0 & 19.2 & 19.2 \\
\hline V & 19.8 & 46.6 & 19.6 & 30.8 & 16.7 & 23.1 & 18.2 & 19.1 \\
\hline VI & 16.9 & 41.5 & 14.4 & 24.5 & 16.3 & 22.5 & 16.1 & 19.6 \\
\hline
\end{tabular}

\begin{tabular}{|c|c|c|c|c|c|c|c|c|}
\hline \multirow[t]{2}{*}{ Nominal size: $10 \%$} & \multicolumn{2}{|c|}{$n=50$} & \multicolumn{2}{|c|}{$n=100$} & \multicolumn{2}{|c|}{$n=200$} & \multicolumn{2}{|c|}{$n=500$} \\
\hline & $\kappa=5$ & $\kappa=20$ & $\kappa=5$ & $\kappa=20$ & $\kappa=5$ & $\kappa=20$ & $\kappa=5$ & $\kappa=20$ \\
\hline I & 35.9 & 45.6 & 33.8 & 39.8 & 29.8 & 35.4 & 32.7 & 32.6 \\
\hline II & 29.6 & 52.3 & 28.7 & 42.0 & 30.0 & 35.6 & 29.1 & 31.8 \\
\hline III & 29.8 & 57.8 & 32.3 & 37.2 & 29.8 & 32.2 & 27.7 & 28.2 \\
\hline IV & 30.5 & 46.0 & 32.2 & 36.4 & 31.4 & 31.4 & 30.8 & 32.4 \\
\hline V & 31.1 & 59.7 & 30.8 & 45.2 & 29.5 & 36.2 & 29.4 & 32.0 \\
\hline VI & 29.1 & 56.2 & 26.9 & 39.0 & 27.3 & 35.4 & 30.2 & 32.2 \\
\hline
\end{tabular}

Nominal size: $20 \% \quad n=50 \quad n=100 \quad n=200 \quad n=500$

$\kappa=5 \quad \kappa=20 \quad \kappa=5 \quad \kappa=20 \quad \kappa=5 \quad \kappa=20 \quad \kappa=5 \quad \kappa=20$

\begin{tabular}{lllllllll} 
I & 52.6 & 63.8 & 51.7 & 55.5 & 49.9 & 55.4 & 52.1 & 52.0 \\
II & 48.4 & 68.8 & 47.7 & 60.1 & 47.9 & 55.1 & 49.8 & 51.3 \\
III & 48.0 & 72.2 & 50.2 & 57.9 & 50.0 & 50.2 & 49.6 & 46.3 \\
IV & 48.4 & 61.4 & 51.1 & 54.8 & 50.1 & 49.3 & 50.4 & 50.1 \\
V & $\mathbf{5 0 . 0}$ & $\mathbf{7 5 . 2}$ & $\mathbf{5 1 . 8}$ & $\mathbf{6 4 . 3}$ & $\mathbf{4 9 . 0}$ & $\mathbf{5 5 . 0}$ & $\mathbf{4 8 . 1}$ & $\mathbf{5 0 . 8}$ \\
VI & $\mathbf{4 7 . 6}$ & $\mathbf{7 0 . 8}$ & $\mathbf{4 6 . 5}$ & $\mathbf{5 5 . 8}$ & $\mathbf{4 8 . 3}$ & $\mathbf{5 4 . 8}$ & $\mathbf{4 9 . 3}$ & $\mathbf{4 9 . 2}$ \\
\hline
\end{tabular}


Table 4: Critical values for the Wald test $\alpha_{1}=\alpha_{2}=0$ in the auxiliary regression

$$
y_{t}=\mu+\alpha_{1} \cos \left(\frac{2 \pi t}{\kappa}\right)+\alpha_{2} \sin \left(\frac{2 \pi t}{\kappa}\right)+u_{t} .
$$

The DGP is a white noise process. The sample size is 1000 . The cycle period $\kappa$ is drawn from a uniform distribution on $[5,20]$.

\begin{tabular}{ll}
\hline Size & Critical value \\
& \\
\hline \hline & \\
$1 \%$ & 12.17 \\
$2.5 \%$ & 10.27 \\
$5 \%$ & 8.90 \\
$10 \%$ & 7.29 \\
$20 \%$ & 5.62 \\
\hline
\end{tabular}


Table 5: The empirical rejection frequency of the Wald test for $\alpha_{1}=\alpha_{2}=0$ in the auxiliary regression

$$
y_{t}=\mu+\alpha_{1} \cos \left(\frac{2 \pi t}{\kappa}\right)+\alpha_{2} \sin \left(\frac{2 \pi t}{\kappa}\right)+u_{t}
$$

when the DGP is

$$
y_{t}=\mu+\beta \cos \left(\frac{2 \pi t}{\kappa}\right)+\beta \sin \left(\frac{2 \pi t}{\kappa}\right)+\varepsilon_{t}
$$

with $\kappa=5$ or $\kappa=20$, while these and other values are taken as starting values in the

\begin{tabular}{|c|c|c|c|c|c|c|c|c|}
\hline \multirow[t]{2}{*}{ DGP } & \multirow[t]{2}{*}{ Sample size } & \multicolumn{7}{|c|}{ Starting value for $\kappa$ (if true $\kappa$ is 5 ) } \\
\hline & & 3 & 4 & 4.5 & 5 & 5.5 & 6 & 7 \\
\hline \multirow[t]{4}{*}{$\beta=1$} & 50 & 0.2 & 1.4 & 15.3 & 100 & 57.5 & 19.4 & 4.0 \\
\hline & 100 & 0.1 & 0.4 & 2.3 & 100 & 10.1 & 0.8 & 0.2 \\
\hline & 200 & 0.1 & 0.1 & 0.6 & 100 & 0.6 & 0.1 & 0.1 \\
\hline & 500 & 0.0 & 0.0 & 0.2 & 100 & 0.2 & 0.0 & 0.0 \\
\hline \multirow[t]{4}{*}{$\beta=10$} & 50 & 0.0 & 0.0 & 0.0 & 100 & 99.3 & 0.6 & 24.1 \\
\hline & 100 & 0.0 & 0.3 & 0.0 & 100 & 10.9 & 0.0 & 0.0 \\
\hline & 200 & 0.0 & 0.2 & 0.0 & 100 & 0.0 & 0.0 & 0.0 \\
\hline & 500 & 0.0 & 0.0 & 0.0 & 100 & 0.0 & 0.0 & 0.0 \\
\hline
\end{tabular}
NLS routine and $\beta$ is either 1 or 10 . The critical values in Table 4 are used.

Starting value for $\kappa$ (if true $\kappa$ is 20 )

$\begin{array}{lllllll}15 & 18 & 19 & 20 & 21 & 22 & 25\end{array}$

$\begin{array}{lllllllll}\beta=1 & 50 & 78.5 & 97.7 & 98.9 & 100 & 100 & 99.8 & 95.6 \\ & 100 & 7.8 & 93.8 & 99.1 & 100 & 100 & 96.6 & 57.3 \\ & 200 & 1.7 & 47.1 & 96.9 & 100 & 97.5 & 75.3 & 21.8 \\ & 500 & 0.0 & 13.5 & 96.8 & 100 & 74.8 & 20.7 & 1.4\end{array}$

$\begin{array}{lllllllll}\beta=10 & 50 & 100 & 100 & 100 & 100 & 100 & 100 & 100 \\ & 100 & 82.6 & 100 & 100 & 100 & 100 & 100 & 74.9 \\ & 200 & 0.5 & 40.1 & 100 & 100 & 100 & 94.8 & 34.5 \\ & 500 & 0.0 & 75.1 & 96.8 & 100 & 72.5 & 0.7 & 13.6\end{array}$


Figure 1: The distribution of the Wald test when $\kappa$ is unknown

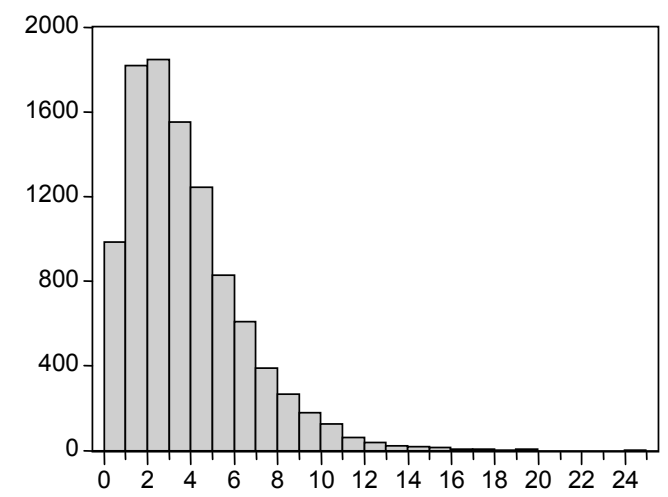

